\title{
Programas de eliminación del Amianto. Lecciones desde Polonia
}

\section{Programs for Asbestos Abatement. Lessons from Poland}

\section{Vega García López}

${ }^{1}$ Instituto de salud pública y laboral de Navarra, Pamplona, España.

Fechas · Dates

Recibido: 2020.11.11

Aceptado: 2021.01.18

Publicado: 2021.01.21
Correspondencia · Corresponding Author

Vega García López

vgarcial@navarra.es;vegagl55@gmail.com 


\section{Resumen}

La comercialización del amianto en Europa de la segunda mitad del siglo XX supuso un consumo de millones de toneladas.

La exposición laboral se ha controlado a partir de la Directiva de 2009 y, en la actualidad, mediante vigilancia epidemiológica, se registran las patologías, mesoteliomas fundamentalmente, por exposiciones pasadas.

Después de prohibida su utilización, aún permanecen cantidades ingentes en edificios, infraestructuras y vehículos, entre otros. El camino hacia su eliminación se inició con una Resolución del Parlamento Europeo, de 2013 y el Dictamen del Comité Económico y Social Europeo (2015/C 251/03).

Con el objetivo de conocer las dificultades de estos planes se ha revisado el de Polonia, único país que hasta la fecha, ha implementado un plan de acción con un gran respaldo financiero y las actuaciones llevadas a cabo en relación con la exposición a amianto en España $y$, Navarra en concreto por contar con un registro exhaustivo de trabajadores expuestos.

El enorme esfuerzo económico que precisan estos planes y los riesgos medioambientales que suponen, merecen una precisa planificación, que exige conocer el no alcance hasta la fecha actual de los objetivos planteados en Polonia, país referente.

Palabras clave: amianto; exposición riesgos ambientales; consenso.

\section{Abstract}

The commercialization of asbestos in Europe in the second half of the 20th century translated into consumption of millions of tons of this material.

Occupational exposure to asbestos is controlled under the 2009 European Union Directive. Currently, through epidemiological surveillance and pathology registries (mainly mesotheliomas), it is possible to record past exposures.

Despite prohibiting its use, large amounts of asbestos remain in buildings, infrastructures and vehicles, among others. The road to elimination of existing asbestos began with a 2013 European Parliament Resolution and the Opinion of the European Economic and Social Committee (2015 / C 251/03)

To better understand barriers to implementing these plans, we reviewed the experience in Poland the only country that to date has implemented an action plan with great financial support, together with actions carried out in Spain generally, and Navarre specifically, given the latter's exhaustive registry of exposed workers.

The enormous economic effort required to implement these plans, along with the environmental risks associated with asbestos abatement, require detailed planning, which should consider understanding why the objectives set by Poland, a benchmark country, have not been achieved to date.

Key words: asbestos; environmental exposure; consensus. 


\section{Introducción}

El término amianto designa a los silicatos fibrosos comprendidos en el registro de sustancias químicas del Chemical Abstract Service (CAS)(1).

Debido a sus propiedades de resistencia, baja conductividad térmica y resistencia química ha sido utilizado ampliamente tanto en construcción (tubos y tejados de fibrocemento, fundamentalmente) como aislante (equipos de protección personal, industria del automóvil, siderúrgica...) y útiles domésticos (tablas de planchar, estufas, secadores...). La comercialización se inició en la segunda mitad del siglo XIX pero su mayor demanda en Europa, Norte América y Australia se produjo en los años, 60, 70 y 80 del siglo XX. Esto hizo que las cantidades que se estiman de consumo durante esos años pudieron llegar a 174 millones de toneladas hasta el año $2000^{(2)}$. En Europa los principales importadores fueron UK, Alemania, Francia e Italia.

En relación a la exposición laboral, todos los países de la, hasta ahora, EU-28 han transpuesto a sus legislaciones nacionales la conocida como Directiva del amianto (Directive 2009/148/EC) ${ }^{(3)}$ han reforzado su implantación con Programas nacionales y Campañas, dirigidas a detectar y controlar materiales que contienen amianto en las construcciones, elaborar planes de gestión de riesgo para la eliminación de materiales, velar sobre la información a los trabajadores expuestos y a la detección de enfermedades relacionadas. Es de destacar el gran impacto sufrido por Italia por enfermedades relacionadas con el amianto y ha desarrollado un "Registro Nacional de Mesoteliomas (ReNaM)"(4) que lleva a cabo una vigilancia epidemiológica exhaustiva de la enfermedad paralela a un gran programa de prevención.

Aunque hace décadas que se fue prohibiendo su utilización: en el entorno, Dinamarca fue el primer país en prohibirlo (1972), Italia en 1992, España en 2001, después muchos otros, entre ellos USA (2002) y toda la EU el 1 de enero de 2005 (Directiva 76/769/CEE), debido al gran período de latencia de la enfermedad (hasta 40 años), no va a ser posible evaluar su efecto en la salud poblacional (mesotelioma, cáncer de pulmón, asbestosis y otros cánceres relacionados (intestinal, ovario, laringe...) en muchos años. Muy al contrario, asistimos en el momento anual al incremento de casos debido a exposiciones de los años 70, 80 y 90 del pasado siglo.

Otro problema, además, es que aunque prohibida su utilización, puede encontrarse en buques, trenes, maquinaria, túneles, tuberías de distribución de agua y cubiertas de edificación.

El camino hacia su eliminación se inició con una Resolución del Parlamento Europeo, de 14 de marzo de $2013^{(5)}$ y, en 2015, el Dictamen del Comité Económico y Social Europeo sobre Erradicar el amianto en la UE (2015/C 251/03)(6) exhortó a la Comisión Europea y a los Estados miembros para desarrollar planes de acción para la eliminación segura del amianto, empezando por los edificios públicos y a nivel local. 
Señalaba expresamente que, hasta esa fecha, solo Polonia había establecido un plan de acción con respaldo financiero con un valor estimado en 10000 millones EUR hasta 2030.

Indicaba además otros ejemplos: En Francia, de un parque de quince millones de viviendas sociales, se han identificado tres millones con problemas relacionados con el amianto y el coste estimado para su necesaria rehabilitación se eleva a 15000 millones EUR. El coste estimado por unidad oscila entre los 15000 y los 20000 EUR. Habría que vigilar atentamente las acciones contra el amianto en la icónica torre Montparnasse de París.

En el Reino Unido está en marcha una campaña para erradicar el amianto en todas las escuelas. Una de las razones para la campaña es el aumento de la tasa de mesotelioma entre los profesores del Reino Unido.

En Lituania, en 2012 dio comienzo un programa de sustitución de los tejados de amianto emprendido por el Ministerio de Agricultura.

Señala la problemática en relación con los residuos, advirtiendo que los vertederos existentes son una solución temporal y evitar el uso de los vertederos de la construcción en general.

Insta a la Comisión Europea a que aprovechara la oportunidad de vincular la retirada segura del amianto a su programa de renovación de los edificios en aras de la eficiencia energética.

La Comisión Europea y los Estados miembros también deberían mejorar la vigilancia del mercado contra la importación a la UE de productos que contengan amianto

La Comisión Europea debería considerar la posibilidad de abrir el acceso a sus Fondos Estructurales explícitamente para los planes de erradicación del amianto.

Indicaba que cualquier plan de acción debe de considerar las competencias de todas las partes interesadas (trabajadores, empresas, coordinadores de seguridad y salud, inspectores de trabajo, asesores, formadores...).

Incidía, además, en el reconocimiento de las enfermedades relacionadas con la exposición a amianto y la mejora de su registro.

Para ello pedía a los Estados miembros que velen por la adecuada formación de los especialistas en medicina del trabajo ser capaces de facilitar la información necesaria a los trabajadores expuestos a amianto que se encuentran bajo su supervisión.

En el 2019 un nuevo Dictamen del Comité Económico y social Europeo "Trabajo con amianto en la renovación energética" Dictamen de iniciativa (2019/C 240/04) (7) considera necesario crear sinergias entre la Directiva relativa a la eficiencia energética de los edificios y la eliminación de sustancias nocivas durante la renovación energética, teniendo en cuenta puntos importantes como garantizarse el número suficiente de vertederos para tratar los residuos, ayuda financiera a los propietarios y adecuadas condiciones de seguridad y salud a los trabajadores. 
A continuación se revisan las actuaciones llevadas a cabo hasta la fecha actual y la evaluación del Plan de Polonia, principalmente, a partir de publicaciones de fuentes fidedignas.

\section{Material y Métodos}

Se ha procedido a localizar en las principales bases científicas y legislativas, estudios y documentos que reflejen lo más rigurosamente una evaluación de los Programas, Planes y otras iniciativas para la eliminación del amianto, fundamentalmente de Polonia.

Así mismo se reproducen los datos y evaluaciones que se analizan en los principales documentos publicados, pues no se dispone de datos de fuentes originales. De igual modo se reproducen gráficos e imágenes de dichas publicaciones. Se citan en el apartado resultados al no ser posible una elaboración propia.

Los datos de patología relacionada con la exposición se publican como tasas de incidencia de Mesotelioma Maligno (MM) por 100000 habitantes, fundamentalmente y los trabajadores expuestos como tasa por 100000 trabajadores.

Por parte del autor, no existe ningún conflicto de interés con las consideraciones que se reflejan en el manuscrito.

\section{Resultados}

\section{Situación actual en Polonia}

Polonia es mencionada en la Resolución del Parlamento Europeo, de 14 de marzo de $2013^{(5)}$, para seguir el ejemplo de un plan que pretendía para 2032 la eliminación del amianto de todos los edificios públicos y concienciar a los propietarios privados $^{(8)}$.

Entre los antiguos países del bloque soviético, Polonia asistió a una auténtica explosión después de la II Guerra Mundial en la reconstrucción, sobre todo, entre los años 70 y 90 hasta su prohibición en 1997. Hay regiones en Polonia como Lublin, región rural, en la que el $90 \%$ de las cubiertas son de fibrocemento(9). La cantidad estimada en cubiertas fue de 738068000 m2 (8.2 millones de toneladas). Y la cantidad de productos con fibrocemento por persona, de media $202 \mathrm{~kg} /$ persona, y puede oscilar de $423 \mathrm{~kg}$ a $100 \mathrm{~kg}$, según regiones.

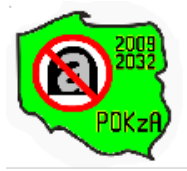

En el Programa para la eliminación del Asbesto en Polonia 2009-2032, se estimaba que aún había 14.5 millones de toneladas de productos con amianto en Polonia 
y para implementar el Programa para el período 2009-2032 se estimaba un coste total de 40.4 billion PLN (zloty), (10 000 millones EUR).

En relación a la patología relacionada con la exposición a amianto, Polonia y otros países del Este de Europa tienen las tasas más bajas de incidencia de mesotelioma maligno (MM), no obstante parece ser un problema de infraestimación de los Registros de cáncer ${ }^{(10)}$.

Existen, además, diferencias entre provincias de Polonia, en 3 se encontraron tasas por encima de 11 casos /100 000 hab. Y, aunque se ha visto correlación positiva entre la concentración de fibras en el aire y la superficie estimada de cubiertas de fibrocemento, no se han encontrado relación significativa entre las tasas registradas de MM y la estimación de productos de fibrocemento en la zona.

En relación a las tasas de MM por género, por regiones se dan mayores tasas en mujeres en la región de Szczucin, que cuenta con una fábrica de fibrocemento(11,12).

\section{Evaluación del Programa}

Desde la aprobación del Programa Integral de eliminación del amianto se describen a continuación los siguientes hechos con los que se concluye su evaluación hasta el momento actual(13) y se citan las siguientes dificultades:

- Falta de comunicación de presencia de asbesto en los edificios por parte de los propietarios a la autoridad correspondiente (se estima que solo se ha registrado la 1/3 parte). Los datos se registran en la "national database bazaazbestova.gov.pl"

- El propietario del edificio, obra... es el responsable de valorar el estado del material (Regulation of the Minister of Economy, Labour and Social Policy, 2004)

- Alto coste del transporte especial de los materiales retirados

- Alto coste de retirada, sobre todo de cubiertas

- Financiación insuficiente. Solo se financia la retirada de material que contiene fibrocemento no la nueva cubierta.

- Falta de motivación ciudadana (los granjeros no están dispuestos a cambiar cubiertas baratas y que sirven para el fin que necesitan por otras más caras pero de las que no ven el beneficio en la salud).

- La ciudadanía solo tiene información de casos de enfermedad a las personas que han trabajado directamente con el material y hace mucho tiempo.

- Hay desigualdad en la cofinanciación de los diferentes áreas ("voivodeship") Las tareas son llevadas a cabo por gobiernos municipales. Hay regiones que no han participado en todas las ediciones del Programa...

- Otro problema es la disponibilidad de vertederos para sustancias peligrosas (hasta el momento, se habían clausurado 17 y solo se han planificado 2 nue- 
vos). Por lo que son totalmente insuficientes. Experiencias como en Italia, han demostrado la existencia de vertederos ilegales ${ }^{(14)}$ o bajo escasa regulación.

- Han surgido muchas dudas de quien tiene que financiar el Programa (Ministerio de Economia, los propietarios, instituciones de protección del medio ambiente ("the National Fund for Environmental Protection and Water Management and the provincial environmental protection funds").

El Programa asumía que de los 14.5 millones de toneladas se iba a disminuir de $2009-2012$ un $28 \%$ de (4 millones t), otro 35\% de 2012-2022 y hasta 2032 el otro $37 \%$.

Los datos que se disponen hasta el momento(15) indican que para Junio de 2017 incluso la cantidad planificada para 2009-2012 no ha sido completada y con gran variación ente las "voivodeships" (un voivodeship es un área administrada por un voivode (gobernador) en varios países del centro y este de Europa).

La evaluación del Programa con información hasta 2017 concluye que no ha sido implementado según los objetivos previstos y se tiene la impresión de que no se conseguirá para el 2032 si se sigue el ritmo actual. Figura 1.

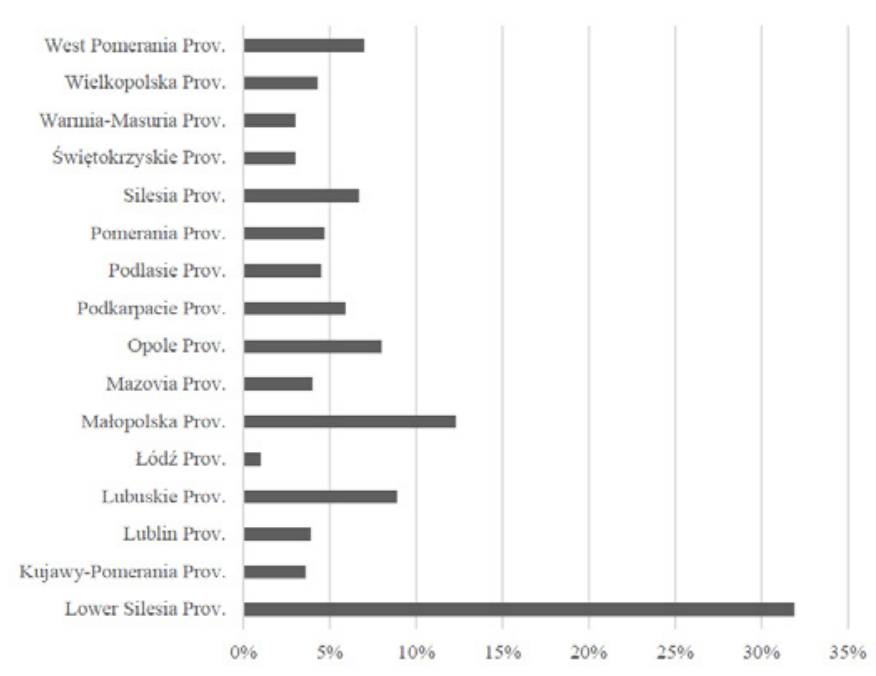

Figura 1: Porcentaje por provincias de productos eliminados que contienen amianto(15)

\section{Situación en España, y Navarra en concreto}

En la totalidad de CCAA se han adoptado medidas para llevar a cabo la normativa vigente del amianto en España, si bien a diferente ritmo en la década anterior(16).

En algunas están además en sus Planes estratégicos, otras han llevado a cabo campañas específicas con pequeñas variaciones entre CCAA.

Las principales actividades realizadas han sido:

- Formación Identificación Materiales 
- Formación gestión de materiales y residuos

- Procedimiento alta empresas RERA (Registro de Empresas con Riesgo de Amianto)

- Comunicación Trabajadores expuestos

- Coordinación vigilancia postocupacional

- Seguimiento de la actividad de vigilancia de la salud postocupacional

- Elaboración Planes Amianto Apoyo

- Análisis cualitativo de fibras y acreditación para el recuento de fibras

- Control del desarrollo y aplicación del RD 396/2006 de 31 de marzo sobre trabajos con riesgo de amianto

- Tramitar e informar los Planes de Trabajo con riesgo de amianto y el Registro de Empresas con Riesgo de Amianto (RERA).

- Asesorar a empresas y particulares sobre procedimientos administrativos y técnicos relativos a materiales con amianto.

- Control del desarrollo y aplicación del RD 396/2006, de 31 de marzo sobre trabajos con riesgo de amianto en cuanto a fichas de vigilancia sanitaria de los trabajadores expuestos

- Programa de Vigilancia de la Salud de los Trabajadores que han estado Expuestos al Amianto (PIVISTEA), solicitadas por la Dirección General de Salud Pública del Ministerio de Sanidad, Convenios INSS reconocimiento EEPP (enfermedades profesionales).

En cuanto a la patología detectada relacionada con la exposición a amianto cabe resaltar los siguientes hechos:

El principal período de importación de amianto en España fue de los años 60 a 80 del pasado siglo. Actualmente en el conjunto de las CCAA hay registrados 56373 (17 645 expuestos, 30387 postexpuestos y 8 341bajas), con diversa distribución geográfica (PIVISTEA 2016).

El número correspondiente a Navarra supone la tasa mayor de expuestos (442/100 000 habitantes) seguida de Pais Vasco (341/100 000 habitantes) y muy a distancia de otras CCAA.

El incremento de muertes por mesotelioma empezó a registrarse los años 80 y posiblemente se prolonguen aun un par de décadas.

Existen diferencias entre CCAA en la Incidencia de Patologías relacionadas con la exposición a amianto en Registros de mortalidad. Según datos del INE basados en la mortalidad por mesotelioma, a lo largo del periodo 2000-2015 el cociente hombre/mujer más bajo (1.5) correspondió a los 140 fallecidos de Navarra. 


\section{Discusión}

La evaluación de la situación actual en Polonia, país pionero en planes de eliminación total del amianto, es de gran utilidad en el momento actual que muchas regiones y países pretenden acometerlos.

La gran cantidad de productos con amianto utilizados en este país, fundamentalmente cubiertas de fibrocemento permite dimensionar, quizás maximizar, el problema al que nos enfrentamos.

Si bien, la falta de exhaustividad de los registros de patología relacionada con la exposición, fundamentalmente mesotelioma maligno, no permite utilizar ésta como un buen indicador de exposición ambiental en este país, el hecho de encontrar mayores tasas en mujeres en regiones con fábricas de fibrocemento, hace coherente los hallazgos.

En cuanto a las dificultades encontradas, es de gran interés conocer los problemas que las administraciones correspondientes van a tener que enfrentarse: de comunicación con los propietarios, de valoración del estado de los materiales, del alto coste de la retirada, de la disponibilidad de vertederos y de las diferencias regionales.

Con respecto a la situación en España se constata el importante abordaje en estas últimas décadas de la exposición laboral, aunque con diferencias entre las CCAA hay implementados en todas ellas Planes estratégicos con importantes avances en la prevención de la exposición de los trabajadores, mediante la formación, la prevención y la vigilancia de la salud.

Los datos de patología registrada mediante el Programa de Vigilancia de la Salud de los Trabajadores que han estado Expuestos al Amianto (PIVISTEA) y los convenios con Seguridad Social, han hecho posible el avance en el reconocimiento como EEPP. En este tema se van acortando las diferencias regionales y los registros pioneros y más exhaustivos, como el de Navarra, permiten concluir que en nuestro país la exposición ha sido fundamentalmente laboral o ha tenido relación con este medio (como la exposición doméstica por trabajo irregular o lavado de ropa).

Para interpretar las diferencias observadas entre CCAA en la Incidencia de patologías relacionadas con la exposición a amianto según género en Registros de mortalidad, hay que tener en cuenta que un cociente hombre/mujer por debajo de 2, se estima que la exposición ambiental es importante. En un estudio reciente, en Dinamarca, la ratio hombre/mujer en regiones de exposición principalmente laboral son altas, en la región de Aalborg de 13:1 $1^{(17)}$ a diferencia de otras regiones en las que ha podido existir exposición ambiental por residir en la proximidad de fábricas ${ }^{(18-21)}$.

En relación a la mayor ratio observada en Navarra hay que considerar que:

- en Navarra (área de Pamplona), se trabajó con lana de amianto en las casas por parte de muchas mujeres para una fábrica de hilatura de amianto en la Ro- 
chapea, y, además, las expuestas laboralmente también suponen un número importante (PIVISTEA).

- Navarra cuenta con el Registro de trabajadores expuestos a amianto más exhaustivo del conjunto de CCAA de España y la tasa mayor de expuestos (442/100 000 habitantes).

- el contacto doméstico por lavar la ropa de los trabajadores varones (importante efectivo).

- El hecho de mantener un sistema de vigilancia de mesotelioma laboral hace que se diagnostiquen más casos no laborales también.

\section{A modo de Conclusión}

Aunque el estudio se limita a la revisión de fuentes secundarias, informes y trabajos publicados, en el no acceso a datos de fuentes originales no hay indicios que hagan sospechar que no se han reflejado fielmente las dificultades encontradas y se admite, con honestidad, que no se van a alcanzar los objetivos fijados inicialmente.

Es necesario compartir esta información con la población y, en concreto, el hecho que la patología detectada en nuestro país se debe fundamentalmente a una exposición laboral que tuvo lugar, fundamentalmente, en la década de los 70 y 80 .

Pero también es una realidad, la cantidad ingente material que queda en construcciones, instalaciones... teniendo en cuenta que los materiales con amianto instalados antes de su prohibición, y siempre que estén en buen estado y no presenten riesgo de liberación de fibras de amianto al ambiente, siguen estando permitidos hasta el final de su vida útil o su eliminación.

Por lo que es de gran interés conocer la experiencia de Polonia, primer país en acometerlo, para cuando se diseñen los Planes de Acción en las regiones y países donde se están aprobando y no solo limitarse a la estimación, entre otras, de la superficie de cubiertas. Las tecnologías actuales permiten calcular con relativa facilidad esta superficie, pero el principal problema es abordar una gestión integral de los materiales que se retiren (para evitar eliminación descontrolada que supondría mayor exposición ambiental) y, fundamentalmente, la importante financiación, en el que se ha estancado, incluso el país modelo, Polonia.

Como problema añadido, es necesario mantener un sistema de alerta frente a la importación de productos que contengan amianto fabricados en otros países donde aún no está prohibido su uso(22).

\section{Bibliografía}

1. Real Decreto 396/2006, disposiciones mínimas de seguridad y salud aplicables a los trabajos con riesgo de exposición al amianto. BOE núm. 86, 11/04/2006. 
2. Vogel L, ETUI. Hesa Newsletter, $n^{\circ} 27$, June 2005 with a special report on Asbestos in the world. 1 de junio de 2005;2005.

3. Directive 2009/148/EC of the European Parliament and of the Council of 30 November 2009 on the protection of workers from the risks related to exposure to asbestos at work. OJ L 330, 16.12.2009

4. Registro Nazionale dei Mesoteliomi Ricerca INAIL, Istituto Nazionale Assicurazione contro gli Infortuni sul Lavoro, Dipartimento di Medicina, Epidemiologia, Igiene del Lavoro ed Ambientale. Disponible en: https://www.inail.it/cs/internet/docs/ ucm_207055.pdf

5. Resolución del Parlamento Europeo, de 14 de marzo de 2013, sobre los riesgos para la salud en el lugar de trabajo relacionados con el amianto y perspectivas de eliminación de todo el amianto existente [2012/2065(INI)].

6. Dictamen del Comité Económico y Social Europeo sobre sobre «Erradicar el amianto en la UE» (2015/C 251/03) 31.7.2015 ES Diario Oficial de la Unión Europea C 251/13.

7. Dictamen del Comité Económico y Social Europeo sobre «Trabajo con amianto en la renovación energética» (2019/C 240/04) 16.7.2019 ES Diario Oficial de la Unión Europea C 240/15.

8. Ministry of Economy (2010) Program for Asbestos Abatement in Poland for 2009-2032. Disponible en: https://www.bazaazbestowa.gov.pl/images/do-pobrania/PROGRAM_ENG.pdf

9. Buczaj A, Brzana W, Tarasińska J, Buczaj M, Choina P. Study on the concentration of airbone respirable asbestos fibres in rural areas of the Lublin region in south-east Poland. Annals of Agricultural and Environmental Medicine [Internet]. 2014;21(3):639-43. Disponible en: http://dx.doi.org/10.5604/12321966.1120617

10. Bianchi C, Bianchi T. Global mesothelioma epidemic: Trend and features. Indian J Occup Environ Med [Internet]. 2014 [citado 9 de marzo de 2020];18(2):82. Disponible en: http://www.ijoem.com/text.asp?2014/18/2/82/146897

11. Kotela I, Bednarenko M, Wilk-Frańczuk M, Kotela P, Wołowiec B, Laskowicz $\mathrm{K}$. [The effects of environmental exposure to asbestos dust on health]. Prz Lek. 2010;67(2):107-9.

12. Krówczyńska M, Wilk E. Asbestos Exposure and the Mesothelioma Incidence in Poland. IJERPH [Internet]. 13 de agosto de 2018 [citado 9 de marzo de 2020];15(8):1741. Disponible en: http://www.mdpi.com/1660-4601/15/8/1741

13. Bernaciak A, Bernaciak A. The efficiency of systemic solutions for the removal of asbestos in Poland - current status and prospects. Economic and Environmental Studies. 1 de diciembre de 2016;16:787-801.

14. Paglietti F, Malinconico S, della Staffa BC, Bellagamba S, De Simone P. Classification and management of asbestos-containing waste: European legislation and the Italian experience. Waste Management [Internet]. 1 de abril de 
2016;50:130-50. Disponible en: http://www.sciencedirect.com/science/article/pii/ S0956053X16300629

15. Szymańska D, Lewandowska A. Disposal of asbestos and products containing asbestos in Poland. J Mater Cycles Waste Manag [Internet]. marzo de 2019 [citado 9 de marzo de 2020];21(2):345-55. Disponible en: http://link.springer.com/10.1007/ s10163-018-0796-4

16. García Gómez M, Castañeda R, García López V, Martínez Vidal M, Villanueva V, Elvira Espinosa M. Evaluation of the national health surveillance program of workers previously exposed to asbestos in Spain (2008). Gaceta Sanitaria [Internet]. enero de 2012 [citado 9 de marzo de 2020];26(1):45-50. Disponible en: https://linkinghub.elsevier.com/retrieve/pii/S0213911111002822

17. Panou V, Vyberg M, Meristoudis C, Hansen J, Bøgsted M, Omland $\varnothing$, et al. Non-occupational exposure to asbestos is the main cause of malignant mesothelioma in women in North Jutland, Denmark. Scand J Work Environ Health [Internet]. enero de 2019 [citado 9 de marzo de 2020];45(1):82-9. Disponible en: http:// www.sjweh.fi/show_abstract.php?abstract_id $=3756$

18. ReNaM Working Group, Corfiati M, Scarselli A, Binazzi A, Di Marzio D, Verardo $M$, et al. Epidemiological patterns of asbestos exposure and spatial clusters of incident cases of malignant mesothelioma from the Italian national registry. BMC Cancer [Internet]. diciembre de 2015 [citado 9 de marzo de 2020];15(1):286. Disponible en: http://bmccancer.biomedcentral.com/articles/10.1186/s12885-0151301-2

19. Musti M, Pollice A, Cavone D, Dragonieri S, Bilancia M. The relationship between malignant mesothelioma and an asbestos cement plant environmental risk: a spatial case-control study in the city of Bari (Italy). Int Arch Occup Environ Health [Internet]. marzo de 2009 [citado 9 de marzo de 2020];82(4):489-97. Disponible en: http://link.springer.com/10.1007/s00420-008-0358-5

20. Magnani C, Dalmasso P, Biggeri A, Ivaldi C, Mirabelli D, Terracini B. Increased risk of malignant mesothelioma of the pleura after residential or domestic exposure to asbestos: a case-control study in Casale Monferrato, Italy. Environmental Health Perspectives [Internet]. septiembre de 2001 [citado 9 de marzo de 2020];109(9):915-9. Disponible en: https://ehp.niehs.nih.gov/doi/10.1289/ ehp.01109915

21. Kurumatani N, Kumagai S. Mapping the Risk of Mesothelioma Due to Neighborhood Asbestos Exposure. Am J Respir Crit Care Med [Internet]. 15 de septiembre de 2008 [citado 9 de marzo de 2020];178(6):624-9. Disponible en: http://www. atsjournals.org/doi/abs/10.1164/rccm.200801-0630C

22. García Gómez M, Alonso Urreta I, Antón Tomey C, Bosque Peralta I, García-Gutierrez MJ, Luna Lacarta FJ, Martínez Arguisuelas N, Mena Marín ML, Vázquez Cortizo M. La coordinación administrativa en salud laboral a propósito de un caso: Zapatas de freno con amianto en una empresa. Rev Esp Salud Pública.2018;92:10 de abril e201804004. 